\title{
La educación universitaria desde la perspectiva del proceso histórico del
} desarrollo

\section{College education from the perspective of the history of the development process}

\author{
Denny D. Granado Castillo \\ dennyd1008@hotmail.com
}

Universidad Politécnica Territorial de Yaracuy "Arístides Bastidas"

Recibido: $15 / 07 / 2017$

Aprobado: 05/10/2017

\section{RESUMEN}

Este estudio tiene como objetivo develar la evolución histórica del desarrollo en el contexto universal para el análisis de sus contradicciones y la propuesta de la orientación de la educación universitaria hacia la liberación del pensamiento. Se ubica en la modalidad de investigación documental. La técnica utilizada fue la revisión de fuentes bibliográficas y el análisis de contenido. Se presentan perspectivas de los autores: Marx (1857) y Freire (1969) y se reflexiona analíticamente con el fin de proponer los basamentos de un nuevo modelo educativo emancipador. Una consideración final relevante es que se debe descolonizar el conocimiento y pensamiento humano (Dussel, 2016), siendo así necesario develar las políticas de desarrollo y sistemas económicos impuestos por los poderes hegemónicos que conciben al capitalismo como la única forma posible de subsistir. Se tiene entonces que superar esas prácticas sociales mediante una Educación liberadora que genere una revolución cultural.

Palabras claves: Educación universitaria, desarrollo, teorías de desarrollo, emancipación, proceso histórico. 


\author{
CIENCIAMATRIA \\ Revista Interdisciplinaria de Humanidades, Educación, Ciencia y Tecnología \\ Año V. Vol. V. №9. Julio - Diciembre 2019 \\ Hecho el depósito de ley: pp201602FA4721 \\ ISSN-L: 2542-3029; ISSN: 2610-802X \\ Universidad Nacional Experimental Francisco de Miranda (UNEFM). Santa Ana de Coro. Venezuela \\ Denny D. Granado Castillo
}

\begin{abstract}
This study aims to unveil the historical evolution of development in the universal context for the analysis of its contradictions and the proposal of the orientation of university education towards the liberation of thought. It is located in the form of documentary research. The technique used was the revision of bibliographic sources and content analysis. The authors' perspectives are presented: Marx (1857) and Freire (1969) and is analyzed analytically in order to propose the foundations of a new emancipatory educational model. A relevant final consideration is that human knowledge and thought must be decolonized (Dussel, 2016), so it is necessary to unveil the development policies and economic systems imposed by the hegemonic powers that conceive of capitalism as the only possible way of subsisting. One has to overcome these social practices through a liberating Education that generates a cultural revolution.
\end{abstract}

Keywords: University education, development, theories of development, emancipation, historical process.

\title{
INTRODUCCIÓN
}

Los procesos de transformación de la sociedad y los cambios surgidos históricamente obligan a indagar, analizar, reflexionar y evaluar la gestión de las políticas públicas en el ámbito de la educación universitaria, desde el surgimiento y las épocas que han marcado la búsqueda de lo denominado como desarrollo pero que en realidad debe ser concebido como el progreso alcanzado en lo social, económico y político de las naciones, con especial énfasis la erradicación de la pobreza, el hambre, los problemas de salud, el analfabetismo y el bajo rendimiento en los niveles del sistema educativo venezolano, al igual que, la degradación del ambiente y la violación de los derechos humanos, tal como lo refiere Moreno (2011). Estas políticas públicas a nivel mundial y en particular en América Latina y en Venezuela, son las que tienen dependencia del Estado, a quien le corresponde establecer las directrices y los procesos para formular e implementar las mismas y han sido influenciadas significativamente por postulados de corrientes del pensamiento y teorías del liberalismo, neoliberalismo, intervencionismo, entre otros. 


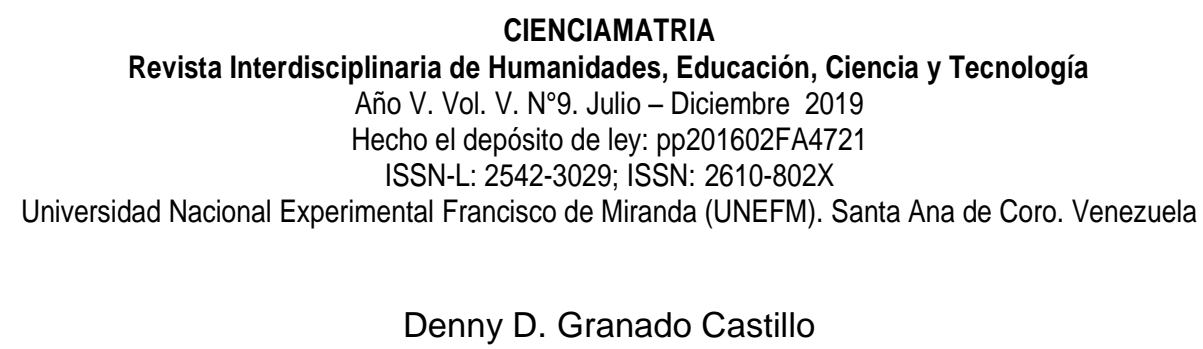

El presente estudio tiene como objetivo develar la evolución histórica del desarrollo en el contexto universal para el análisis de sus contradicciones y la propuesta de la orientación de la educación universitaria fundamentada en la liberación del pensamiento propuesta por Freire (1969). Esta investigación es de tipo documental, bajo enfoque cualitativo. Se utilizó la técnica de la revisión de fuentes bibliográficas y el análisis de contenido, referido por Abela (2001). Se presentan las distintas perspectivas de los autores: Max (1857), Freire (1969), Rist (1997) y Salas (2016) para profundizar en la temática del desarrollo y el análisis crítico de su incidencia en la Educación; se reflexiona analíticamente con el fin de proponer los basamentos para un nuevo modelo educativo emancipador.

\section{Educación Universitaria y el Proceso Histórico del Desarrollo}

En el ámbito académico el accionar en la búsqueda del progreso social debe ser asumido con espíritu de lucha, humildad, ideal de venezolanidad, con el afán y el compromiso de contribuir en la reconstrucción del auge social y económico de la Patria Grande, lo cual se sustenta en la conjugación de los saberes que permean las ciencias para el desarrollo estratégico y que sirve de base al proceso transformador del hombre desde el ser, el saber, el hacer y el convivir. De esta manera se podrá retribuir a la nación con los aportes teóricos oportunos a los cambios requeridos, la consolidación de la identidad, la cultura, la educación orientada al desarrollo integral del ser social.

La Educación Universitaria como proceso fundamental en el desarrollo de la sociedad y en el reimpulso de la liberación de los pueblos, debe ser orientada hacia la educación emancipadora de los actores sociales que asumen los retos con participación protagónica en el proceso educativo de transformación social en los procesos de lucha. Desde esta perspectiva se debe teorizar sobre la teoría de la liberación, reflexionar sobre la Pedagogía del Oprimido instituida por Paulo Freire (1969). Esta necesidad de educar para liberar el pensamiento crítico reflexivo se fundamenta en el "Análisis 


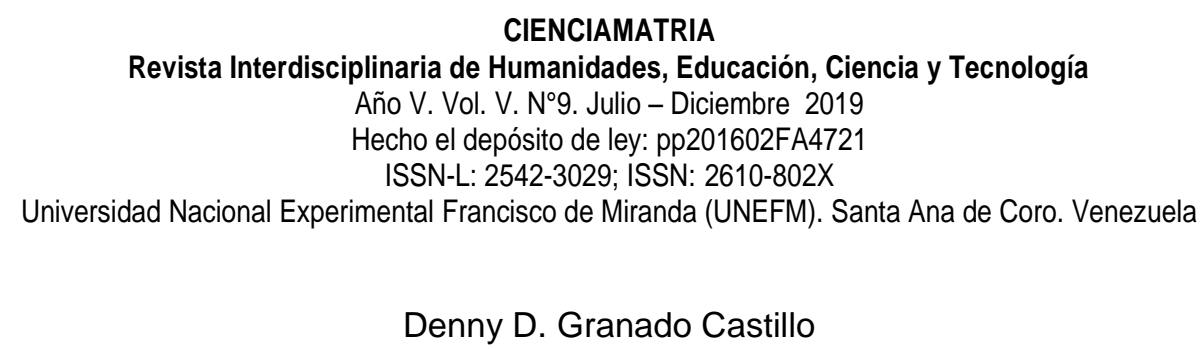

coyuntural de la situación política y económica del país", que ha heredado del proceso del desarrollo una crisis social inducida por las políticas neoliberales y capitalistas de los imperios internacionales, siendo la Educación el arma para combatir estas desigualdades e impulsar el desarrollo humano, social, cultural, político y económico de nuestros pueblos, tal como se proyecta en la Constitución de la República Bolivariana de Venezuela.

Tras la búsqueda de esta educación para la emancipación del hombre, surge la interrogante: ¿Cuál es la evolución histórica del desarrollo en el contexto universal para el análisis de sus contradicciones en el marco de la orientación de la Educación Universitaria fundamentada en la liberación del pensamiento?

\section{Objetivo General}

Develar la evolución histórica del desarrollo en el contexto universal para el análisis de sus contradicciones y la propuesta de la orientación de la educación universitaria fundamentada en la liberación del pensamiento.

Para el abordaje de la investigación surge el planteamiento de indagar sobre los elementos que fundamenten la intencionalidad de cómo conducir la educación hacia una concepción emancipadora, y abocarse a entender lo definido como proceso de desarrollo y todo lo que ello envuelve, para lo cual se hace necesario revisar, analizar, cuestionar y reflexionar los preceptos contenidos en la epistemología, teorías, enfoques y modelos sobre el desarrollo, que permiten conocer la esencia del progreso de los pueblos en diversos ámbitos, visibilizar los elementos y argumentos que determinan cómo abordar esa semántica para la comprensión de las realidades en tiempos pasados y en el presente, de allí que puedan abordarse los siguientes aspectos: 


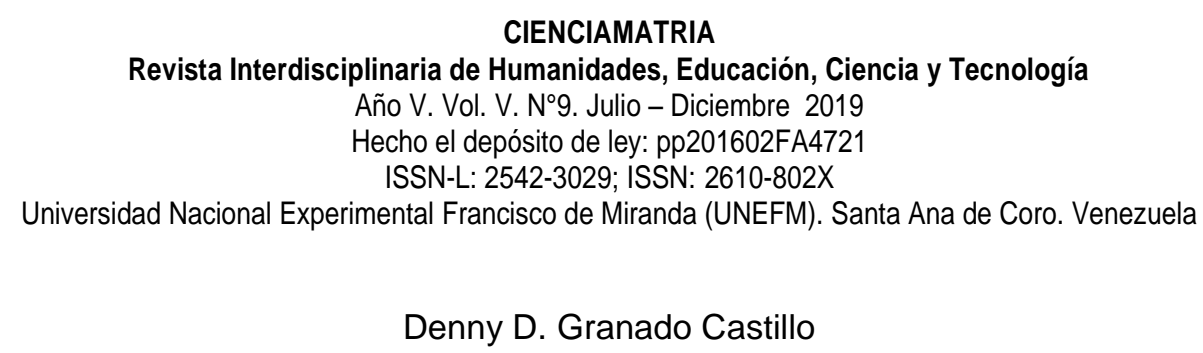

\section{Las Armas Deshonestas del Capitalismo para Idear el Discurso del Desarrollo}

Desde esta perspectiva, el análisis sobre la idea del desarrollo lleva a pensar que el proceso histórico ha permitido al hombre experimentar en todas las etapas de su evolución valerse de las ideas y creencias impuestas desde costumbres, las formas de pensar, de actuar, las formas de cambiar, las maneras de relacionarnos con la naturaleza de nuestros nativos ancestrales, y las formas de producir. En este sentido, el ser humano se ha visto envuelto por el arma de la seducción, tal como lo plantea Rist Gilbert (1997) en The History of Developmet, sobre el discurso del "desarrollo" que es dependiente de su poder para seducir, encantar, cautivar, fascinar, así como también de su atropello para desviarse de la realidad y mentir. Sobre esta base, se puede decir que no es fácil contrarrestar esa noción de desarrollo porque de ella nadie se queja, nadie habla mal, por lo cual es difícil no verse hipnotizado por el discurso manipulador que utiliza como estrategia, con el uso de ideas seductoras y promesas sobredimensionadas, con capacidad para la administración de las expectativas y aspiraciones de una amplia masa de la población para justificar el velo que oculta la verdad y la realidad tangible.

El uso de la falacia utilizada en el discurso del progreso, ha llevado a pensar que si no se está en sintonía de los avances de formación academicista, técnico científico y de la era tecnológica, puedes llegar a ser un fracasado. El poder hegemónico impuesto ha enquistado los antivalores inyectados por la guerra de cuarta generación, para controlar nuestra manera de accionar, lo cual causa desestabilización emocional con la intencionalidad de generar crisis y cambiar el patrón cultural.

Evaluar esos aspectos permite determinar que aunque no es nada fácil, para contrarrestar ese sistema de dominación del capitalismo moderno, se debe emprender la lucha ideológica con el pueblo a través de la educación como instrumento para modificar la conducta del ser humano. 


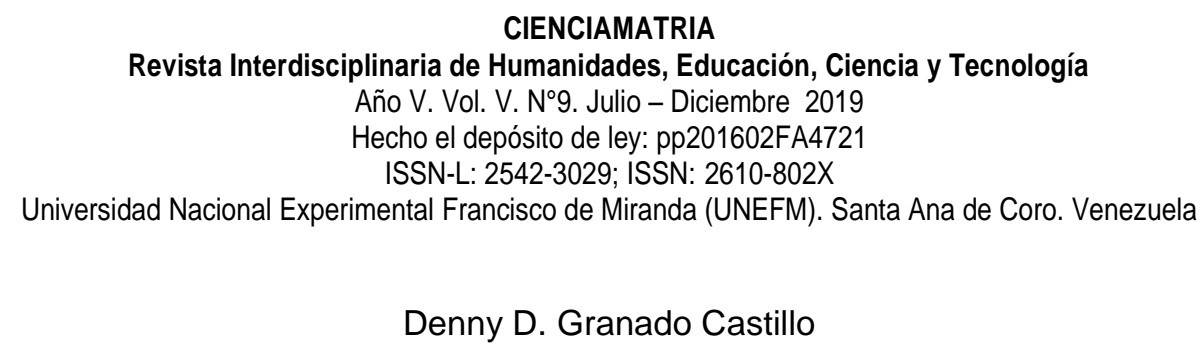

¿Qué hacer para despertar conciencia?, generar propuestas donde se involucre el pueblo y fundamentarlas con el debate y confrontación de ideas, el análisis histórico de los procesos para hacer memoria y valorar la praxis equívoca impuesta por el capitalismo, es decir seguir el precepto de Karl Marx (1957) sobre la dialéctica y la reconstrucción de la realidad. Esto nos debe permitir establecer cimientos para desmontar ese discurso del desarrollo, romper con esas políticas de dominación y liberar el pensamiento del pueblo y del hombre en búsqueda de liberar la sociedad y establecer teorías de educar para el trabajo productivo e independizarse de la educación para el empleo.

\section{Las teorías del desarrollo y el impulso figurado de la transformación social}

Al abordar las teorías del desarrollo y hablar del impulso figurado de la transformación social, se debe asumir la postura reflexiva para comprender y determinar la corresponsabilidad del individuo y de los poderes establecidos en el fortalecimiento socioeconómico de nuestros pueblos. En este sentido, es importante considerar la concepción que pueda tener un individuo de su vida en relación a los elementos fundamentales que contribuyan al buen vivir de la humanidad.

Una de las maneras de explicar estas ideas o concepciones que asume el individuo es reflexionar sobre lo propuesto por Rodríguez (2009) sobre teoría clásica planteada por Smith, Ricardo, Malthus, Mills, en la cual se señala de modo general que la riqueza se genera del trabajo, la división de éste y la libre competencia, así como también plantean el establecimiento de normas que regulen la distribución de la economía, pues asumen la postura de que el crecimiento económico es un factor fundamental del bienestar social.

Por otra parte, es importante resaltar que, al hablar de economía política, podemos señalar lo planteado por Marx (1957) quien considera que, el hombre en su afán de organizar para producir las distintas maneras, establece relaciones como individuo con 


\section{Denny D. Granado Castillo}

la sociedad, lo que origina la conformación de las estructuras sociales, políticas, jurídicas, entre otros. Marx al hablar de los problemas de tipo económico, señala a las necesidades económicas y sus relaciones con el resto de vida circundante, entre los aspectos abordados emplea el análisis de la mercancía, el trabajo, el valor y el dinero, pero enfatiza en la lucha de las clases sociales y la justicia social.

Esto sirvió de base para los postulados de los pensadores modernos y justificar sus propuestas, así como también a lo planeado por Keynes (1965) con su intencionalidad particular, incentivar el control de la economía en periodos de crisis, con una supuesta visión global del empleo, el interés y el dinero, y con el uso de la estrategia de maniobra e intervención del Estado en la política tributaria y monetaria.

Puede entenderse entonces que, esas premisas teóricas clásicas presentan limitaciones en sus visiones, razón por la cual debemos buscar un conocimiento alternativo e innovador que abra espacios para la discusión y que permita transitar en sentido diferente al recorrido por el conocimiento convencional, y despojarse de toda la influencia del colonialismo y el neoliberalismo económico. Todo ello ha causado gran controversia entre los que por un lado apoyan la instrumentación de políticas basadas en estas proposiciones y por otro, los que no las comparten, lo cual obliga a revisar, entender e intentar explicar cuál ha sido el impacto de estas ideas de desarrollo y su aplicación en el ámbito de la administración privada, social y pública.

Sobre la base de lo expuesto anteriormente, puede afirmarse que estas premisas siempre han pretendido explicar y fundamentar las teorías o modelos de desarrollo mediante la utilización de métodos científicos como el uso de estadísticas que propician el escenario para la utilización de terminologías técnicas(como el índice de desarrollo, crecimiento económico, industrialización e indicadores) que hace complicado el entendimiento de los pobladores que no son catalogados como élites científicas e intenta excluir al hombre de pueblo como ente protagónico y corresponsable del bienestar que le permita ir en la búsqueda de un mejor vivir. 


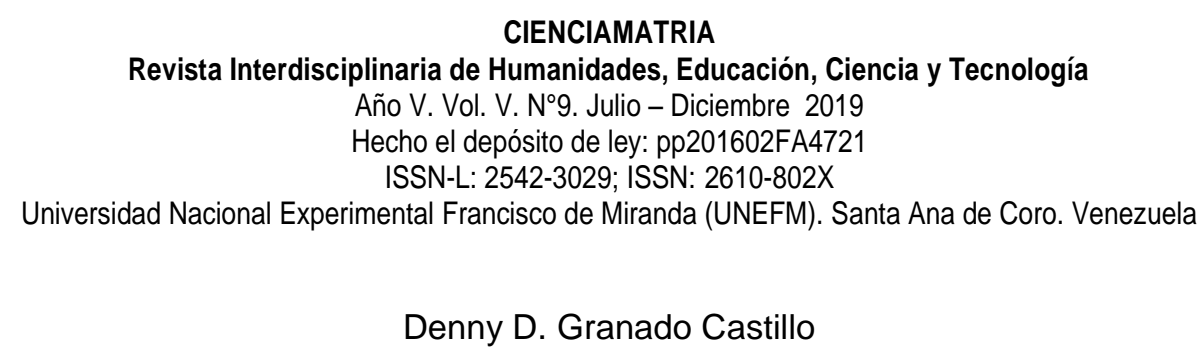

Es importante destacar que debe reflexionarse sobre las clases sociales, tomando en consideración que la sociedad ha cambiado en la evolución histórica del desarrollo, y los actores de este proceso deben tomarse en cuenta en la desconstrucción y en la comprensión de las experiencias que nos impulsen a construir. Esto podrá lograrse al apropiarse de la teoría para emancipar al hombre y reafirmar lo que decía Marx "las circunstancias nos hacen transformarnos y mutarnos, tenemos que ver la realidad más allá de la realidad misma".

En esta línea del pensamiento, cabe destacar el enfoque filosófico de Marx como instrumento de análisis crítico reflexivo de los escenarios y contextos de la sociedad en lo político, lo económico y lo social desde la totalidad concreta, lo cual permitirá elevar los niveles de conciencia e impulsar el proceso transformador de la educación para erradicar el modelo burocrático de estado manipulador del sistema y estructuras sociales instituidas en el país.

\section{La crisis del Fordismo - keynesiano}

Es interesante evaluar lo inmerso en esta temática para la contraposición de ideas y establecer posturas. Se señala entre los aspectos más relevantes de esos momentos históricos que las políticas económicas de Keynes (ob.cit) fundamentadas en la aplicación de un modelo de intervencionismo en el cual el Estado asume de manera más dinámica el control de la economía, conllevaron a lo que se conoce como el estado de bienestar que tenía como objetivo salir de la embarazosa crisis que vivía el sistema capitalista mundial especialmente en los Estados Unidos en el año de 1929.

En el caso de Venezuela esas políticas liberales inciden en la decadencia de la sociedad manipulada por una "burguesía" dependiente del Estado rentista petrolero, que escasamente coloca su capital de riesgos para la inversión y las inventivas tecnológicas, no se aplica una política económica tendiente a resguardar los intereses nacionales, y es marcada la dependencia del gran capital transnacional, creando hábito 


\section{Denny D. Granado Castillo}

especulativo, lo cual devela el carácter usurero de los sectores capitalistas venezolanos.

De allí que, es certera la afirmación de Luis Salas (2016): no vivimos una etapa de inflación, sino de acaparamiento crónico, el cual, se acrecienta debido al poder adquisitivo de la población; aunado al bloqueo internacional, al boicot productivo como sucede actualmente con la Colgate-Palmolive, empresas Inlaca, entre otras, quiénes simplificaron la producción o paralizan el proceso de fabricación; este panorama se agrava por la caída estrepitosa de los ingresos proveniente del estiércol del diablo, asociado a la crisis estructural del capitalista mundial, que al parecer, se profundizará en los próximos meses o años.

Esta burguesía se basa en la promoción ilusoria de desarrollo que envuelve a la población desesperada y desesperanzada, desorientándola y sustituyendo los valores ciudadanos, por el robo y la farsa implementada por la especulación y el acaparamiento, en los cuales se envuelven una serie de cosas donde privan la inseguridad y la escasez, y obligan al ciudadano común a ir en la búsqueda de medios para sobrevivir por encima de los intereses colectivos y ser partícipes en una especie de saqueo organizado que en determinadas épocas ha utilizado como estrategia de querella política el bachaqueo, el cual es la inoculación sistemática de la visión capitalista para estimular el capitalismo de consumo.

Este es el modelo actual o contemporáneo de obtener riqueza temporal y coyuntural, pero de a poco atrofia los valores humanos, familiares y sociales. Esta desviación de principios del hombre ha radicalizado la especulación y el bachaqueo el cual en los actuales momentos de dificultades es el medio utilizado para crear una cultura de que el trabajo productivo no es necesario, no hace falta, lo cual desvirtúa la buena práctica de ciudadanía, tal como lo plantea Salas (ob.cit)

De allí que la reflexión de lo señalado anteriormente lleva a recapacitar y plantearse ¿Qué hacer?, lo cierto es que todos debemos asumir los retos, las responsabilidades 


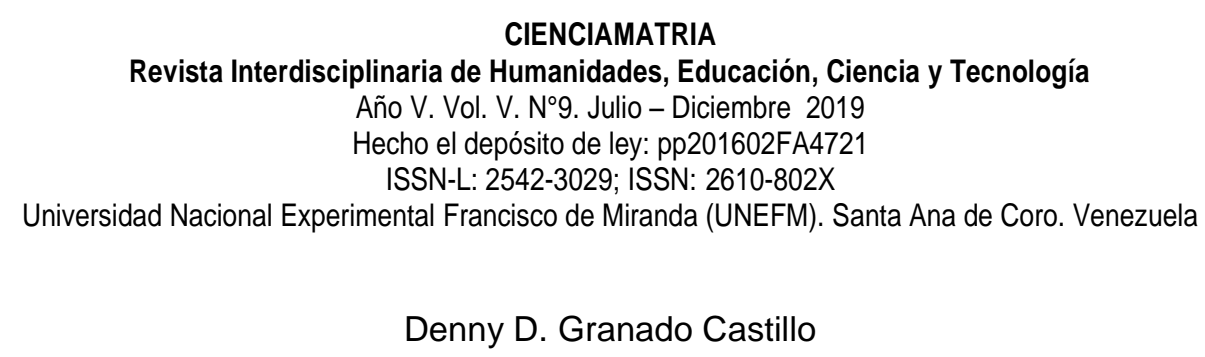

en afrontar la realidad, establecer nuevos modelos fundamentados en la Educación como instrumento para generar el análisis crítico reflexivo y fundar valores socialistas.

\section{La comprensión de la crisis del capitalismo y la decadencia de la modernidad}

El punto de partida de la reflexión es referir lo relacionado a las medidas de ajustes estructurales y la reestructuración capitalista. Esto conduce a evaluar los elementos que han conllevado a las desigualdades sociales, que se evidencian en la acumulación de riquezas ostentadas por un porcentaje minoritario de los 6000 millones de personas que habitan en el globo terráqueo, se tiene así que solo pocos cerca de $20 \%$ de la población tienen la fortuna de apropiarse de la riqueza material (82,7\%) que le pertenece al pueblo, tal como lo plantea Correa (2012).

Sobre la base de lo planteado anteriormente, es oportuno preguntar ¿Porqué estas diferenciaciones en la distribución del capital que acrecienta las disconformidades en la sociedad?, solo en estos tiempos de cambios y de intención de educación liberadora, se presentan escenarios propicios para el debate de ideas y la reconstrucción de políticas que perfilen hacia una gestión pública eficiente, hacia el bienestar social, se podrá facilitar herramientas a los pueblos para liberar el pensamiento y estimular el sentido crítico, lo cual permitirá valorar la realidad, aquella que evidencia que mientras que la gente de pueblo permanece constantemente trabajando en el intento de generar transformaciones sociales, solo muy poquitos cerca del $20 \%$ de la pachamama se repartía el capital económico que era y nos pertenece a todos.

Sólo el reflexionar permite afirmar que esas políticas de economía neoliberal nos han querido dañar, lo que es indudable solo al observar lo que pasa en el mundo y en la realidad que nos rodea.

En ese orden de ideas, es oportuno preguntar ¿Deben establecerse políticas públicas en el sector educativo que permitan establecer una planificación estratégica orientada 


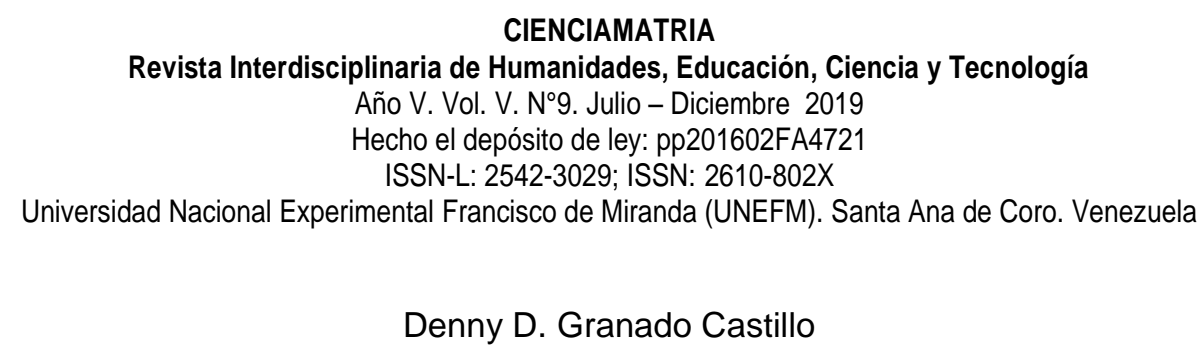

hacia la formación de los estudiantes y futuros profesionales para cultivar en ellos el empoderamiento del sentido crítico, analítico y reflexivo?

En esa línea de pensamiento, se debe propiciar el aprendizaje de los ciudadanos sobre la premisa de valorar que las ofertas engañosas de los poderes políticos y económicos de las cuales la mayoría hemos sido víctimas ingenuas, no llevan por el camino correcto y que esas políticas solo generan las crisis y caos de la nación fingida, la que esconde la verdadera Venezuela, como lo diría Uslar Pietri (1949), la nación infiltrada e impregnada de inconsciencia de la burguesía emergente y de la burocracia gubernamental, ajustada a las fórmulas o normas impuestas por el Fondo Monetario Internacional y el Banco Mundial, esa nación que muestra, que todo al parecer funciona mejor, sin sincerar que al despilfarrar los recursos económicos, la consecuencia es peor.

Esta crisis solo ha dejado como resultado el déficit fiscal y presupuestario, el déficit comercial en el cual las importaciones son mayores que las exportaciones. Además de ello se genera la inflación, entre otros, problemática relevante en el mundo que ha forjado el desigual desarrollo, evidenciado al analizar el esquema que presenta la distribución de la riqueza mundial, que paradójicamente adquiere forma de copa de champagne, la cual nos señala el desbalance de la repartición de los ingresos globales en el planeta, la abundante ganancia para pocos, sin importar la mayor proporción de pobladores.

\section{Hablemos de la década perdida y su influencia en la educación universitaria}

Para el abordaje de esta temática, es necesario realizar un análisis sobre los aspectos que determinan lo que aconteció en la Década Perdida de los años 80 e intentar relacionar lo sucedido con el proceso de involución de la educación en esas épocas pasadas que manipularon y coartaron el pensamiento crítico de los más débiles ideológicamente y tenían la intencionalidad de reprimir a los de los mayor claridad 


\section{Denny D. Granado Castillo}

ideológica. Este análisis permitirá contraponer estos sucesos con la evolución del sistema educativo en estas épocas de cambio y transformación en el siglo XXI, en particular en la Educación Universitaria.

El análisis realizado conduce a exponer que la década de los años 80 fue la etapa de la desesperanza y es allí cuando se comienza a aplicar los planes del neoliberalismo que asolaron a la sociedad venezolana. Sucesos acontecidos a nivel mundial y se reproduce una recomposición del sistema capitalista en la cual la burguesía toma el control.

Esta década marca el estancamiento de los países latinoamericanos con la imposición de políticas que conllevan a una crisis aguda y generalizada que se caracterizó por ser la más grave desde la depresión de los años 30, teniéndose en ella la transición del capitalismo liberal al capitalismo neoliberal, y que podemos considerar con firmeza "una época de pérdida para el desarrollo económico y social de la región" y una desaceleración del mismo, con caída de indicadores macroeconómicos, evidenciada en el descenso de la actividad económica, altos índices de desempleo, disminuye el salario real de los trabajadores, aumento de las tasas de interés, desproporcionado endeudamiento externo, entre otros. Allí se quiebra el capitalismo de producción y surge el capitalismo de consumo, siendo el consumismo una consecuencia de los valores del sistema capitalista.

Como consecuencia de todo lo anterior, se suscita un crecimiento ficticio de la economía, no mejoran los problemas sociales, se impulsa la migración de las personas del campo a la ciudad, lo que genera mayor concentración de la población en las zonas más marginales de condiciones de vida en las ciudades, lo cual agudiza la pobreza, se incrementa la dependencia al observar mayor disponibilidad de acceso de las personas a las entidades bancarias en las cuales se utilizan políticas crediticias (créditos para viviendas, vehículos y tarjetas de créditos) e implantación de sistemas hipotecarios que luego comprometen los bienes y el producto del trabajo de los ciudadanos. 


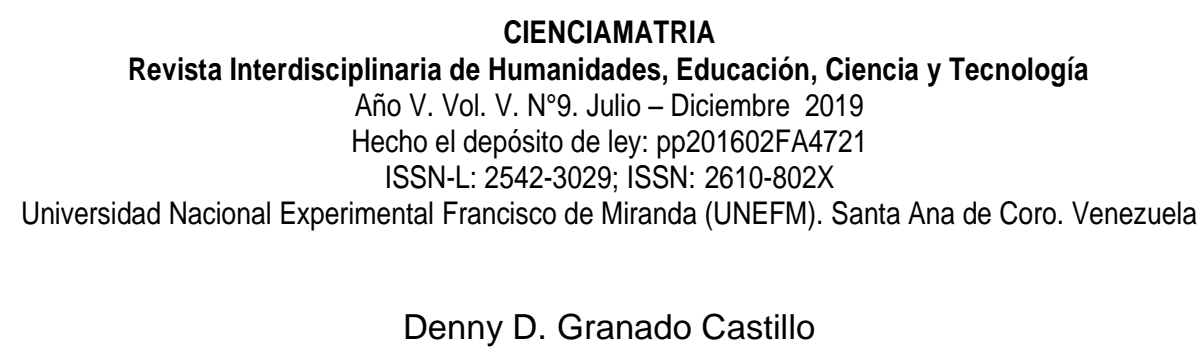

En el campo educativo, puede señalarse la influencia negativa de la década perdida, en la cual se utiliza un modelo de imposición neoliberal atrincherado en los centros del saber y el conocimiento, con sus teorías rígidas de productividad y sus esquemas de acción dependentista, ante la deshumanización paulatina de las instituciones y los vicios que a lo largo de los siglos se han enquistado en el seno de la universidad.

Todo esto aún sigue latente y es evidenciado entre otros ámbitos, en el aula de clases con el desarrollo del currículo oculto que juega al fracaso y al desprestigio de la buena intencionalidad de lo previsto en la Constitución de la República Bolivariana de Venezuela, la Ley Orgánica de Educación, los Programas Nacionales de Formación de la Misión Alma Máter, y el Plan de la Patria, con posturas equívocas, mezquinas, con el uso de las herramientas del enemigo oculto y mediocre que solo pretende con su ignorancia ideológica en algunos casos, pero en otros que se etiquetan cultos en lo político, mantener la hegemonía del poder.

Esto justifica recapacitar y profundizar en la política propuesta por el comandante eterno Hugo Rafael Chávez Frías, y teorizar para generar fundamentos teóricos que sustenten el modelo de horizontalidad que propulse el empoderamiento de los estudiantes y profesionales como agentes activos del poder popular en los procesos de gestión de las instituciones, orientado a desmontar el modelo de acumulación de riquezas a costilla del trabajador y la trabajadora y a promover una educación liberadora dirigida hacia la conquista de libertades sociales.

\section{MATRIZ METODOLOGICA}

El estudio se ubica en la modalidad de investigación documental.. La técnica utilizada fue la revisión de fuentes bibliográficas, electrónicas y el análisis de contenido, según Abela (2001). Como técnicas de recolección de información se utilizó la revisión de fuentes bibliográficas, electrónicas, y el análisis crítico reflexivo del contenido de las fuentes consultadas, según Abela (ob.cit). 


\section{Denny D. Granado Castillo}

\section{RESULTADOS DE LA INVESTIGACIÓN}

Como resultado del análisis crítico reflexivo de la temática abordada surgen los siguientes presupuestos:

Puede afirmarse que el discurso del desarrollo ha manipulado la educación como herramienta de formación, y no ha sido utilizada como instrumento de lucha para gestar la clara visión del hombre como agente social de transformación. Qué papel ha jugado la familia, la escuela, la comunidad, el gobierno, la institucionalidad del Estado, que todos tenemos la responsabilidad de ejecutar lo previsto en la ley de vida, en nuestra carta magna, de instruir, de plantear y ejecutar de manera eficiente los programas, los proyectos, los mecanismos de mitigación, sin cartas bajo la manga, sin disimulos, sin mezquindad, evidenciar la realidad coyuntural que vive Venezuela, que no es aislada de lo que ocurre en la geopolítica internacional que muestra panoramas semejantes, mas desalentadores y más drásticos.

Esto lleva a reflexionar; seremos capaces de comprender lo que nuestros antepasados pudieron afrontar, de vivir con restricciones, de preservar y entender que muchos procesos se puede ejecutar con más independencia de la tecnología, de no caer en las trampas del desarrollo, del capitalismo mercantilista, del consumismo, de la complicidad que favorece al protegido por los poderes económicos. $Y$ actuar con responsabilidad, con compromiso, con menor agresividad en la vida, al ambiente, al planeta, mostrar la disponibilidad de prepararse para educar de manera objetiva a mis semejantes, de compartir el conocimiento, de brindar oportunidades para el cambio de actitud.

Habrá que preguntarse ¿que causa la incertidumbre?, será porque siempre hablamos de miseria, de hambruna, la desigualdad de condiciones de vida de los pueblos designados pobres, desfavorecidos, los que padecen de las inclemencias de los fenómenos naturales y los inducidos por la actividad y la inconsciencia humana, catástrofes como el cambio climático, que genera daños irreversibles como incendios, desforestación, sequía, cambio de comportamiento de los seres vivos, la vegetación, 


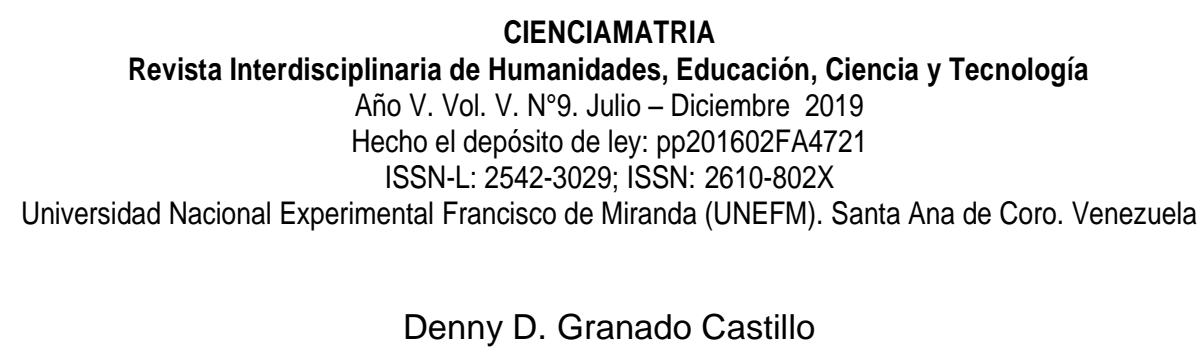

los animales, pérdida de la biodiversidad, migración de las especies por efecto de los fenómenos antropogénicos.

Debemos reflexionar sobre lo señalado y asumir el reto de concienciar y propiciar espacios para debatir y cuestionar el caos, la guerra económica, los efectos del cambio climático en el planeta, educar para afrontar el desafío climático del siglo XXI e internalizar lo que plantea Sven Lindqvist "Lo que nos falta es el valor para comprender lo que sabemos y sacar conclusiones de ello".

Mediante este instrumento de liberación del pensamiento, de la confrontación de ideas se podrá dar herramientas para entender la necesidad de aplicar medidas de mitigación de los efectos de la acción del hombre causadas a la madre tierra. En este sentido, (López Echegarai, 2019, p. 85) plantea que "El éxito de cualquier política de sustentabilidad, necesita de la participación social de todos los actores de la comunidad universitaria".

Así que el desafío es asumir la educación como la base para la transformación del ser humano y de los pueblos, instrumento para propulsar el diálogo y la conjugación de los saberes, del conocimiento, lo cual hace al hombre ser más sensible, más humano, más actor clave para impulsar el cambio de hábitos y de conducta.

\section{CONSIDERACIONES FINALES}

La revisión y el análisis de lo planteado por diversos autores sobre el discurso del desarrollo, permitió realizar un abordaje comparativo y reflexivo para develar los preceptos que envuelven el proceso del desarrollo y su influencia en la educación durante varias décadas en la sociedad mundial y especialmente en nuestra América.

En el transcurrir de este desafío reflexivo y valorativo de la educación y en especial en el sector universitario, se hace evidente las deficiencias en el sistema educativo que no establece las innovaciones y políticas que puedan impulsar los cambios y evoluciones requeridos para el progreso de los pueblos, sino que por lo contrario se desarrolla 


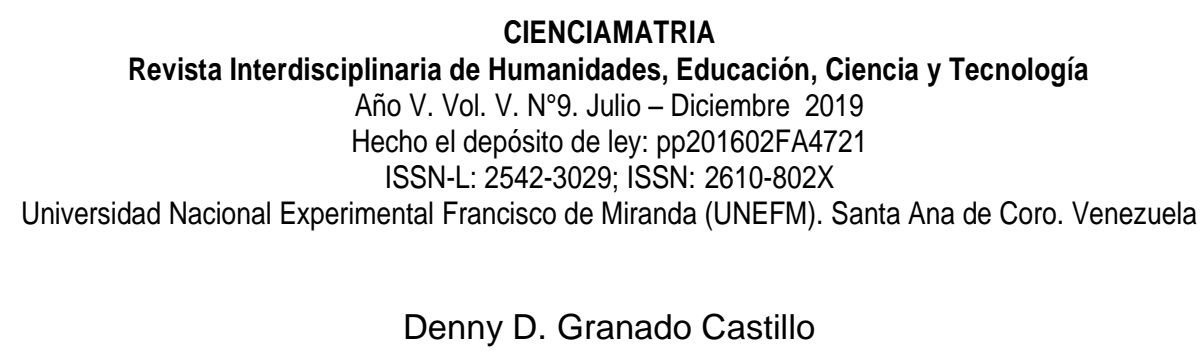

descontextualizado mostrando su desvinculación con los fenómenos de la realidad social. En consecuencia, se hace necesario reorientar la educación como instrumento esencial en el avance de la nación y el buen vivir de la sociedad.

¿Qué se debe hacer? Debemos descolonizar la memoria y el conocimiento, para descolonizar debemos conocer los fundamentos, interpretarlos, develarlos y superarlos, y buscar argumentos para desmontar con nueva propuesta revolucionaria, generar ideas y teorías con sentido crítico que permita proponer alternativas orientadas hacia la transformación social.

Debemos superarlos con nuevas praxis sociales que rompan los esquemas impositivos y retóricos, contrarrestando esos mecanismos de alienación y dominación implementados por el colonialismo aun latente en nuestros tiempos, es decir no debemos practicar las mismas herramientas que lo que hacen es reproducir como si esas prácticas capitalistas fueran la única manera de vivir, valerse de herramientas que no lleven a ratificar, a enquistar esa cultura permeada por principios deshumanizados que entrampa a los seres humanos y no permite desarrollar el pensamiento del hombre. Puede afirmarse que el análisis de los temas abordados permite fijar las postura sobre esas formas políticas impuestas que conciben al capitalismo como la única forma posible de subsistir, utilizando las armas de la seducción, las estrategias de consolidación de las desigualdades sociales con una distribución de la riquezas desproporcionada y el estancamiento social, dominada por los principios del individualismo la competencia y la tasa de ganancia.

El instrumento fundamental para esta superación es la Educación, a través de la cual se busque el rescate de nuestras costumbres y culturas latinoamericanas, en busca de descolonizar la memoria y el pensamiento, y así romper con los esquemas impuestos de represión de la libertad. Por ello puede aseverarse que la educación universitaria del estudiantado y los actores sociales se debe visionar hacia la formación integral basada 


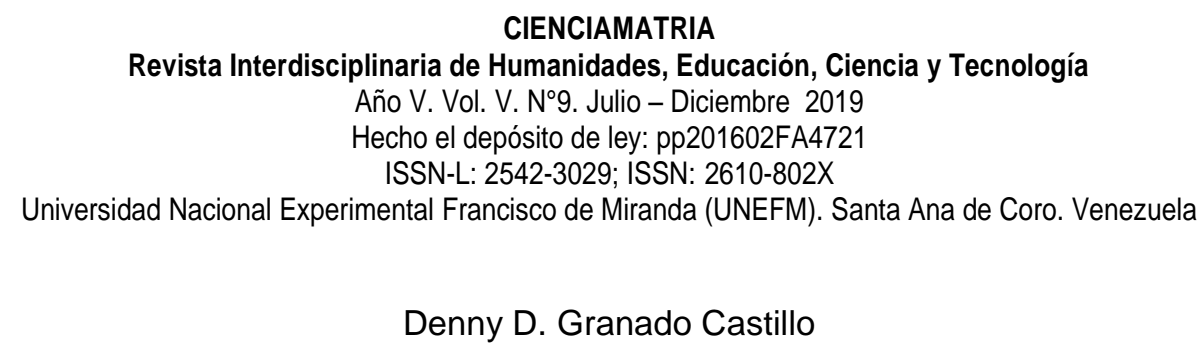

en los principios y preceptos establecidos en Constitución, los planes de desarrollo y las respectivas leyes de la república de Venezuela, para educar para la vida.

Se hace imperante proponer estrategias que generen el despertar conciencia en el estudiante y los actores sociales involucrados en el proceso educativo, perfilando la formación de estos hacia un ser humano que se deslastre de los antivalores y la deshumanización de los procesos, individuos consientes de la necesidad de la reconstrucción del conocimiento producto de la conjugación de los saberes científico y popular, alineados en la búsqueda de la relación armónica del hombre con la naturaleza.

Se requiere un nuevo ser social, que podrá proponerse formarlo mediante la educación emancipadora de los actores partícipes en el proceso de construcción de la Patria Grande, los cuales les corresponde asumir los retos de una verdadera muestra del sentido socialista y revolucionario.

Finalmente, puede considerarse que lo planteado en esta reflexión ha permitido crear la expectativa, conocer los espitemes que debemos superar para poder generar propuestas que desmonten estos preceptos teóricos que se han venido manejando por la ciencia, por ello se propone establecer una nueva manera de hacer ciencia y generar aportes con fundamentos teóricos que puedan ser evaluados y a futuro implementados en la nueva política de la gestión pública de la educación universitaria en Venezuela.

\section{REFERENCIAS CONSULTADAS}

1. Abela, J. (2001). Las técnicas de Análisis de Contenido: Una revisión actualizada. [Documento en línea]. Disponible: http://public.centrodeestudiosandaluces.es/pdfs/S200103.pdf.

2. Constitución de la República Bolivariana de Venezuela. (1999). Gaceta Oficial de República Bolivariana de Venezuela, 36860. (Extraordinario). Diciembre 30,1999. 


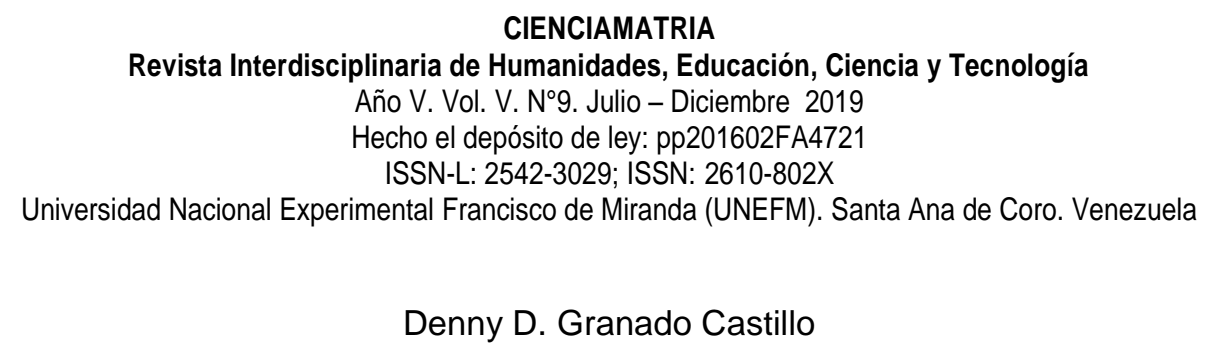

3. Correa (2012). La pobreza se sirve en una copa de Champaña. [Documento en línea]. Disponible: http://www.elespectador.com/noticias/actualidad/vivir/pobrezase-sirve-una-copa-de-champana-articulo-353398.

4. Dussel, E. (2016). Sin una Descolonización del Pensamiento no hay Revolución. [Documento en línea]. Disponible: http://laiguana.tv/articulos/38305-entrevistaenrique-dussel-descolonizacion-revolucion-clodovaldo.

5. Freire, P. (1969) Pedagogía del Oprimido. [Documento en línea]. Disponible: http://www.servicioskoinonia.org/biblioteca/general/FreirePedagogiadelOprimido. pdf

6. Keynes, J. (1965). Teoría general de la ocupación, el interés y el dinero. Fondo de cultura económica. Segunda edición. [Documento en línea]. Disponible: http://www.listinet.com/bibliografia-comuna/Cdu332-38FB.pdf.

7. López Echegarai, M. (2019). Política de sustentabilidad ambiental. Avances en el contexto universitario. Revista Arbitrada Interdisciplinaria Koinonía, 4(7), 59-87. Recuperado de http://fundacionkoinonia.com.ve/ojs/index.php/revistakoinonia/article/view/194/15 8

8. Marx, K. (1857), Elementos Fundamentales para la Crítica de la Economía política (borrador 1857-1858), décima quinta edición, siglo XXI editores, volumen 1, España, p. 21.

9. Moreno, A. (2011): "Políticas Públicas de Educación Universitaria para el desarrollo integral de Venezuela: hacia una aproximación conceptual". Docencia Universitaria, Vol. XII, № 1, Año 2011 SADPRO - UCV. Universidad Central de Venezuela, Caracas. [Documento en línea]. Disponible: http://www.ucv.ve/fileadmin/user_upload/sadpro/Documentos/docencia_vol12_n1 _2011/9_art_5._ana_beatriz_y_graffed.pdf

10. Rist, G. (1997). The History of Developmet. [Documento en línea]. Disponible: https://books.google.co.ve/books?id=NpV64yAbBvQC\&redir_esc=y.

11. Rodríguez, C. (2009). Los Economistas Clásicos. [Documento en línea]. Disponible: http://disertaciondeeconomia.blogspot.com/

12. Salas, L. (2016). La crisis de los 90 fue peor..[Documento en línea]. Disponible: http://www.noticierodigital.com/2016/01/luis-salas-la-crisis-de-los-90-fue-peor/ 


\title{
CIENCIAMATRIA
}

Revista Interdisciplinaria de Humanidades, Educación, Ciencia y Tecnología

Año V. Vol. V. №9. Julio - Diciembre 2019

Hecho el depósito de ley: pp201602FA4721

ISSN-L: 2542-3029; ISSN: 2610-802X

Universidad Nacional Experimental Francisco de Miranda (UNEFM). Santa Ana de Coro. Venezuela

\author{
Denny D. Granado Castillo
}

(C)2019 por los autores. Este artículo es de acceso abierto y distribuido según los términos y condiciones de la licencia Creative Commons Atribución-NoComercial-Compartirlgual 4.0 Internacional (CC BY-NC-SA

4.0) (https://creativecommons.org/licenses/by-nc-sa/4.0/). 